\title{
Computing the broadband vibroacoustic response of arbitrarily thick layered panels by a wave finite element approach
}

\author{
D. Chronopoulos ${ }^{\mathrm{a}, \mathrm{b}}$, M. Ichchou ${ }^{\mathrm{b}}, \mathrm{B}$. Troclet ${ }^{\mathrm{a}, \mathrm{c}}$, O. Bareille ${ }^{\mathrm{b}}$ \\ ${ }^{a}$ EADS Astrium ST, 66 Route de Verneuil, BP3002, 78133 Les Mureaux Cedex, France \\ ${ }^{b}$ Ecole Centrale de Lyon, 36 Avenue Guy de Collongue, 69134 Ecully Cedex, France \\ ${ }^{c}$ Ecole Normale Supérieure de Cachan, 61 Avenue du Président Wilson, 94230 Cachan, France
}

\begin{abstract}
A robust procedure for the prediction of the dynamic response of layered panels within a SEA wave-context approach is proposed hereby. The dispersion characteristics of two dimensional composite orthotropic structures are predicted using a Wave Finite Element method. By manipulating the mass and stiffness matrices of the modelled structural segment a polynomial eigenvalue problem is formed, the solutions of which correspond to the propagation constants of the waves travelling within the structure. The wavenumbers and group velocities for waves comprising out of plane structural displacements can then be calculated. Using the numerically extracted wave propagation data the most important SEA quantities of the structure, namely the modal density and the radiation efficiency of each wave type are calculated. The vibroacoustic response of the structure under a broadband diffused excitation is then computed within a SEA approach. The impact of the symmetric and the antisymmetric vibrational motion of the panel on its sound transmission loss is exhibited and the approach proves robust enough for thin as well as for thick layered structures.
\end{abstract}

Keywords: Aerospace composites, Wave propagation, Sound Transmission Loss

\section{List of Abbreviations}

CLPT Classical Laminate Plate Theory

DoF Degree of Freedom

FE Finite Element

FSDT First-order Shear Deformation Theory

HSDT Higher-order Shear Deformation Theory

MAC Modal Assurance Criterion

Email address: chronopoulos.dimitri@gmail.com (D. Chronopoulos) 

PST
Periodic Structure Theory
SEA
Statistical Energy Analysis
STL
Sound Transmission Loss
WFEM
Wave Finite Element Method

\section{Introduction}

Complex, non-isotropic stratified and sandwich type constructions are widely used in engineering applications such as in the aerospace and automotive industries, mainly because of their high stiffness-to-mass ratio and the fact that their mechanical characteristics can be designed to suit the particular purposes. Unluckily this high stiffness-tomass ratio being responsible for the increased mechanical efficiency, imparts as well efficient acoustic radiation. The modelling of the vibrational behaviour of complex composite structures has been a field of extensive study in modern mechanical engineering. The knowledge of the wave propagation characteristics within a structure seems to provide a key to decode and model its vibrational behaviour.

Analytic formulas for the dispersion characteristics in orthotropic thin plates can be found in classical publications [1, 2] starting with the CLPT [3], developped as an extension of the Kirchhoff-Love's theory for isotropic plates and suitable for thin panels. Furthermore, the FSDT [4] takes into account the transverse shear deformation of the panel and can be used for predicting the dispersion characteristics at higher frequencies. Such classical theories have been successfully used by many authors, as in [5] in order to model the radiation efficiency and the vibroacoustic response under a reverberant field [6] of thin orthotropic panels. Kurtze and Watters [7] were the first to develop an asymptotic model for the wave dispersion into symmetric flat thick sandwich structures. They divided the flexural wave speed of a sandwich panel (frequency-wise) into three sections, the first characterized by the panel vibrating as a whole, the second by the core's shear wave speed and the third by each of the two facesheets vibrating separately and loaded with half of the core mass. Nevertheless, the model assumed the core to be incompressible, and the deformation of the panel in the thickness sense could not be modelled. Dym and Lang [8] were the first to develop a structural model for an infinite sandwich panel by using the kinematic assumptions of [9] and derived the five equations of motion corresponding to the symmetric and antisymmetric motion of the panel. Moore and Lyon [10] extended this structural model to symmetric sandwiches with an orthotropic core. A consistent (HSDT) taking into account the core's shear deformation was developped in [11] and used in [12,13] to construct a structural model of an infinitely long sandwich panel and calculate its vibroacoustic response within an SEA context.

Calculating the wave propagation characteristics of non conformal structures has been a subject of intense research over the past years. The author in [14] presents an approach for computing the wave propagation within hollow beam structures. In [15] the authors predict the dispersion characteristics within composite panels in the framework of a SFE 
approach, overcoming the limitations of a CLPT modelling. In [16], the authors calculated the phase constant surfaces of periodic composite and stiffened structures using FE and PST. The authors gave an expression of the radiation efficiency of the panels based on the calculated wave displacement shapes. The STL of the panels was expressed through the radiation and mechanical impedances of the structures. An insight to the calculation of the same quantities by a modal approach was also given. The authors in $[17,18]$ used a multi-layer analytical model based on Mindlin theory to compute the dispersion characteristics within layered structures. However while the asymmetric mode of motion was correctly captured, the symmetric mode of motion was not naturally expressed using a Reissner-Mindlin's type of displacement field. The same authors presented an approach for taking into account for the symmetric wavw motion for thick panels in [19]. The WFEM was introduced in [20]. Its main underlying assumption is the periodicity of the structure to be modelled. The PST is then coupled to the FEM. Several applications of the WFEM have been conducted to predict the wave speeds of one-dimensional [21, 22] and two-dimensional structures [23]. A set of structures including a vehicle's chassis [24], and a stiffened plate [25, 26] has been modelled using the WFE method. The two-dimensional technique was successfully used in [23] to model the wave propagation in orthotropic thin panels and sandwich structures.

Throughout the applications of the WFEM, simplicity is exhibited as its main advantage compared to other approaches mentioned above. Indeed, it suffices to develop the FE model of a structural segment comprising a small number of DoF and inject the extracted matrices into an ordinary mathematics software. Very fast calculation of the dispersion characteristics for every direction of propagation are observed (see [27]) when using the 2D WFEM as presented in [23]. Moreover, due to the solid FE formulation of the problem the WFEM can predict all the higher order waves propagating within a structure (including symmetric wave motion) without any former assumptions of the displacement unknowns in contrast to the analytical approaches. Furthermore, the 3D elasticity theory which governs the solid FE used results in very accurate results in a broadband frequency range.

In this paper the two-dimensional WFEM is employed in order to compute the STL of thick layered structures by accounting for their symmetric and antisymmetric wave motion. Anisotropic, multi-layered panels can be accurately modelled using this generic approach for a broadband frequency range. The resonant transmission coefficient of the panels is directly expressed in relation to the SEA quantities. A way for computing the reverberant field STL of the structures directly derived by their SEA properties is also exhibited. The results are successfully compared to experimental measurements encountered in the open bibliography.

The paper is organized as follows : In sec.2 the process of solving the resulting polynomial eigenproblem as well as the post-processing of the occurring solutions is discussed. In sec.3 the calculation of the main SEA quantities, namely the modal density and the radiation efficiency for each wave mode of the layered panels is presented. In sec. 4 some numerical applications are exhibited in order to validate the conducted work. The conclusions are eventually given in sec.5. 


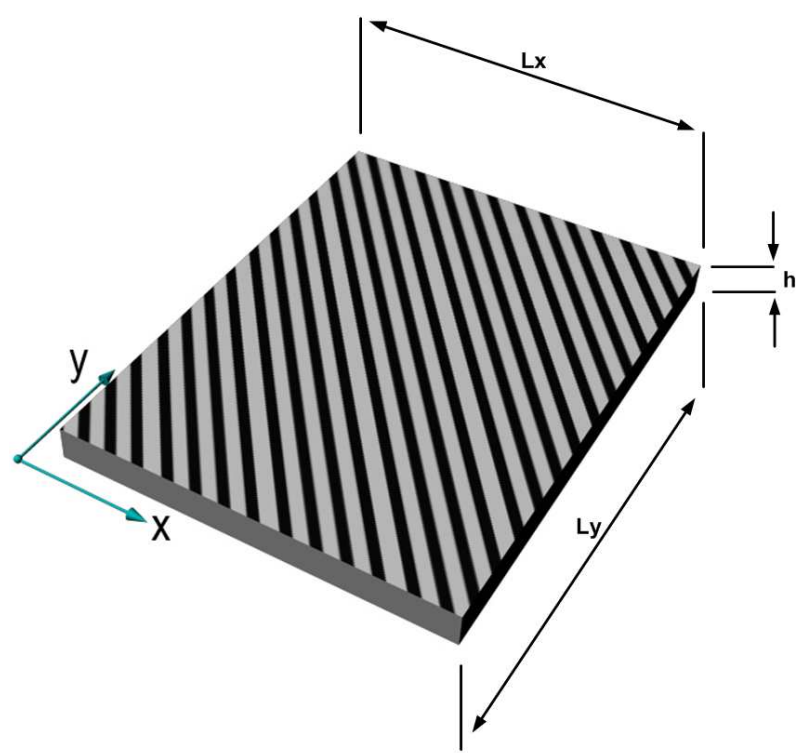

FIGURE 1: A composite panel modelled within the present approach

\section{The two dimensional WFE method}

\subsection{Description of the method}

A rectangular composite panel is considered hereby (see fig.1) with $L_{x}, L_{y}$ its dimensions and $h$ its thickness. A periodic segment of the panel with dimensions $d x$ and $d y$ (see fig.2) is modelled using FE.

Initially, the mass and stiffness and damping matrices of the segment $\mathbf{M} \mathbf{K}$ and $\mathbf{C}$ are extracted using classical FEM algorithms. The entries for each DoF, of every node laying on the same edge of the segment, say edges Q, $\mathrm{R}, \mathrm{S}$ and $\mathrm{T}$, are placed in the mass and stiffness matrices so that the vector of displacements can be written as : $\mathbf{u}=\left\{\mathbf{u}_{\mathbf{Q}} \mathbf{u}_{\mathbf{R}} \mathbf{u}_{\mathbf{S}} \mathbf{u}_{\mathbf{T}}\right\}^{T}$. Following the analysis presented in [23] the time-harmonic equation of motion of the segment assuming uniform and structural damping for all the DoF can be written as :

$$
\left(\mathbf{K}+\mathrm{i} \omega \mathbf{C}-\omega^{2} \mathbf{M}\right) \mathbf{u}=\mathbf{F}
$$

where $\eta$ is the structural damping coefficient, $\omega$ is the angular frequency and $\mathbf{F}$ the vector of the nodal forces. Then the dynamic stiffness matrix can be written as :

$$
\mathbf{D}=\mathbf{K}+\mathrm{i} \omega \mathbf{C}-\omega^{2} \mathbf{M}
$$

therefore eq.(1) may be written as : 


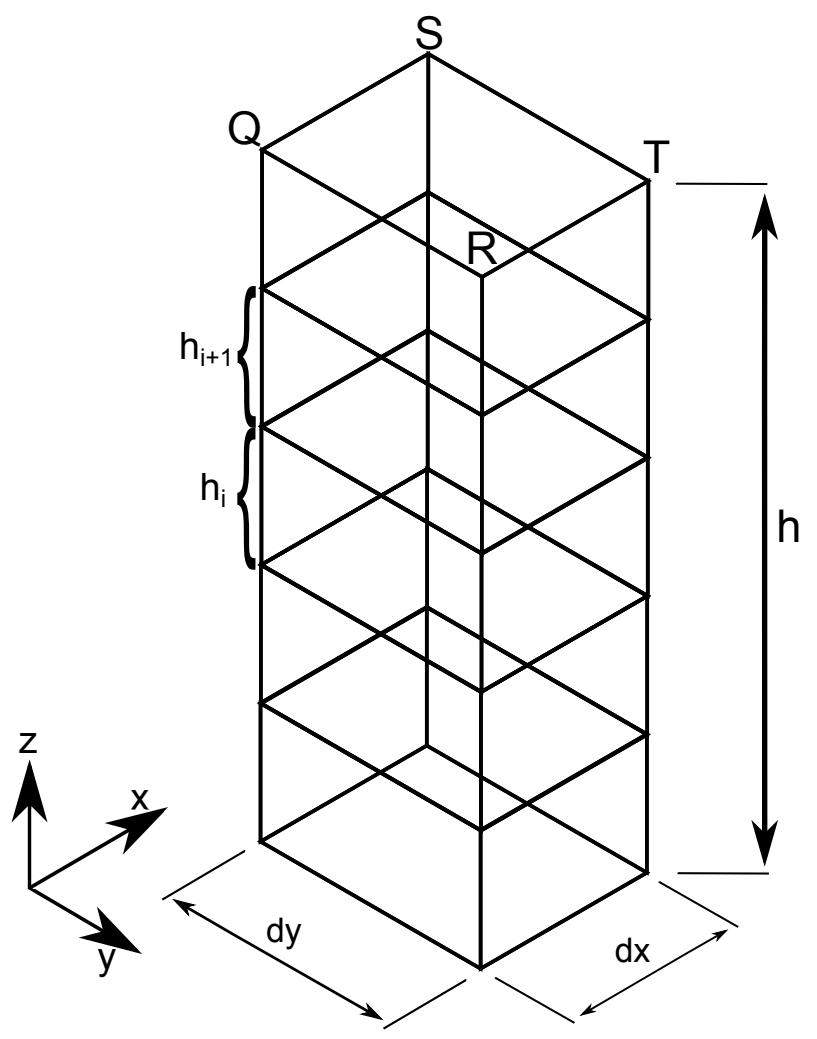

FIGURE 2: View of the modeled periodic segment with its edges Q, R, S and T 


$$
\left[\begin{array}{llll}
\mathbf{D}_{\mathrm{QQ}} & \mathbf{D}_{\mathrm{QR}} & \mathbf{D}_{\mathrm{QS}} & \mathbf{D}_{\mathrm{QT}} \\
\mathbf{D}_{\mathrm{RQ}} & \mathbf{D}_{\mathrm{RR}} & \mathbf{D}_{\mathrm{RS}} & \mathbf{D}_{\mathrm{RT}} \\
\mathbf{D}_{\mathrm{SQ}} & \mathbf{D}_{\mathrm{SR}} & \mathbf{D}_{\mathrm{SS}} & \mathbf{D}_{\mathrm{ST}} \\
\mathbf{D}_{\mathrm{TQ}} & \mathbf{D}_{\mathrm{TR}} & \mathbf{D}_{\mathrm{TS}} & \mathbf{D}_{\mathrm{TT}}
\end{array}\right]\left\{\begin{array}{c}
\mathbf{u}_{\mathrm{Q}} \\
\mathbf{u}_{\mathrm{R}} \\
\mathbf{u}_{\mathrm{S}} \\
\mathbf{u}_{\mathrm{T}}
\end{array}\right\}=\left\{\begin{array}{c}
\mathbf{F}_{\mathrm{Q}} \\
\mathbf{F}_{\mathrm{R}} \\
\mathbf{F}_{\mathrm{S}} \\
\mathbf{F}_{\mathrm{T}}
\end{array}\right\}
$$

Using the Floquet theory for a rectangular segment and assuming a time-harmonic response the displacements of each edge can be written as a function of the displacements at one single edge. Taking edge $Q$ as the edge of reference we have :

$$
\mathbf{u}_{\mathbf{R}}=\lambda_{x} \mathbf{u}_{\mathbf{Q}}, \mathbf{u}_{\mathbf{S}}=\lambda_{y} \mathbf{u}_{\mathbf{Q}}, \mathbf{u}_{\mathbf{T}}=\lambda_{x} \lambda_{y} \mathbf{u}_{\mathbf{Q}}
$$

Using the same theory, the force vectors can be written as :

$$
\mathbf{F}_{\mathbf{R}}=\lambda_{x} \mathbf{F}_{\mathbf{Q}}, \mathbf{F}_{\mathbf{S}}=\lambda_{y} \mathbf{F}_{\mathbf{Q}}, \mathbf{F}_{\mathbf{T}}=\lambda_{x} \lambda_{y} \mathbf{F}_{\mathbf{Q}}
$$

With $\lambda_{x}$ and $\lambda_{y}$ the phase constants which are related to the wavenumbers $k_{x}$ and $k_{y}$ through the relation :

$$
\lambda_{x}=\mathrm{e}^{-i k_{x} d_{x}}, \lambda_{y}=\mathrm{e}^{-i k_{y} d_{y}}
$$

The displacement vector can therefore be written as :

$$
\left\{\begin{array}{c}
\mathbf{u}_{\mathbf{Q}} \\
\mathbf{u}_{\mathbf{R}} \\
\mathbf{u}_{\mathbf{S}} \\
\mathbf{u}_{\mathbf{T}}
\end{array}\right\}=\left\{\begin{array}{c}
\mathbf{I} \\
\lambda_{x} \mathbf{I} \\
\lambda_{y} \mathbf{I} \\
\lambda_{x} \lambda_{y} \mathbf{I}
\end{array}\right\} \mathbf{u}_{\mathbf{Q}}
$$

Assuming no external excitation, equilibrium along edge $\mathrm{Q}$ implies that :

$$
\left\{\begin{array}{llll}
\mathbf{I} & \lambda_{y}^{-1} \mathbf{I} & \lambda_{x}^{-1} \mathbf{I} & \lambda_{x}^{-1} \lambda_{y}^{-1} \mathbf{I}
\end{array}\right\}\left\{\begin{array}{c}
\mathbf{F}_{\mathbf{Q}} \\
\mathbf{F}_{\mathbf{R}} \\
\mathbf{F}_{\mathbf{S}} \\
\mathbf{F}_{\mathbf{T}}
\end{array}\right\}=\mathbf{0}
$$

Eventually, substituting eq.(7,8) in eq.(1) we end up with the eigenproblem :

$$
\left\{\begin{array}{llll}
\mathbf{I} & \lambda_{y}^{-1} \mathbf{I} & \lambda_{x}^{-1} \mathbf{I} & \lambda_{x}^{-1} \lambda_{y}^{-1} \mathbf{I}
\end{array}\right\} \mathbf{D}\left\{\begin{array}{c}
\mathbf{I} \\
\lambda_{x} \mathbf{I} \\
\lambda_{y} \mathbf{I} \\
\lambda_{x} \lambda_{y} \mathbf{I}
\end{array}\right\} \mathbf{u}_{\mathbf{Q}}=\mathbf{0}
$$

which can be written in the form : 


$$
\left(\begin{array}{c}
\left(\mathbf{D}_{\mathbf{Q Q}}+\mathbf{D}_{\mathbf{R R}}+\mathbf{D}_{\mathbf{S S}}+\mathbf{D}_{\mathbf{T T}}\right)+\left(\mathbf{D}_{\mathbf{Q R}}+\mathbf{D}_{\mathbf{S T}}\right) \lambda_{x}+\left(\mathbf{D}_{\mathbf{R Q}}+\mathbf{D}_{\mathbf{T S}}\right) \lambda_{x}^{-1} \\
+\left(\mathbf{D}_{\mathbf{Q S}}+\mathbf{D}_{\mathbf{R T}}\right) \lambda_{y}+\left(\mathbf{D}_{\mathbf{S Q}}+\mathbf{D}_{\mathbf{T R}}\right) \lambda_{y}^{-1}+\mathbf{D}_{\mathbf{Q T}} \lambda_{x} \lambda_{y}+ \\
\mathbf{D}_{\mathbf{T Q}} \lambda_{x}^{-1} \lambda_{y}^{-1}+\mathbf{D}_{\mathbf{S R}} \lambda_{x} \lambda_{y}^{-1}+\mathbf{D}_{\mathbf{R S}} \lambda_{x}^{-1} \lambda_{y}
\end{array}\right) \mathbf{u}_{\mathbf{Q}}=\mathbf{0}
$$

Various methods exist for the solution of the eigenproblem and are discussed extensively in [16, 23]. In this paper the scenario in which the frequency and the wavenumber towards $y$ direction are considered as fixed will be adopted. For each set of fixed $\omega, k_{y}$ the entirety of $k_{x}$ values are sought and values for intermediate $\omega, k_{x}$ and $k_{y}$ can be found by interpolating on the known results. For a set of fixed $\omega, k_{y}$ the non-linear eigenproblem of eq.(10) is reduced to :

$$
\left(\mathbf{A}_{2} \lambda_{x}^{2}+\mathbf{A}_{\mathbf{1}} \lambda_{x}+\mathbf{A}_{\mathbf{0}}\right) \mathbf{u}_{\mathbf{Q}}=\mathbf{0}
$$

where :

$$
\mathbf{A}_{\mathbf{i}}=\left\{\begin{array}{l}
\mathbf{D}_{\mathbf{Q T}} \lambda_{y}^{2}+\left(\mathbf{D}_{\mathbf{Q R}}+\mathbf{D}_{\mathbf{S T}}\right) \lambda_{y}+\mathbf{D}_{\mathbf{S R}}, \mathrm{i}=2 \\
\left(\mathbf{D}_{\mathbf{Q Q}}+\mathbf{D}_{\mathbf{R R}}+\mathbf{D}_{\mathbf{S S}}+\mathbf{D}_{\mathbf{T T}}+\mathbf{D}_{\mathbf{Q S}}+\mathbf{D}_{\mathbf{R T}}\right) \lambda_{y}+\mathbf{D}_{\mathbf{S Q}}+\mathbf{D}_{\mathbf{T R}}, \mathrm{i}=1 \\
\mathbf{D}_{\mathbf{R S}} \lambda_{y}^{2}+\left(\mathbf{D}_{\mathbf{R Q}}+\mathbf{D}_{\mathbf{T S}}\right) \lambda_{y}+\mathbf{D}_{\mathbf{T Q}}, \mathrm{i}=0
\end{array}\right.
$$

The above quadratic eigenproblem can also be converted as shown in [28] into an ordinary linear generalized eigenproblem of twice the size, by defining a new vector $\mathbf{z}=\lambda_{y} \mathbf{u}_{\mathbf{Q}}$ :

$$
\left[\begin{array}{cc}
-\mathbf{A}_{\mathbf{0}} & \mathbf{0} \\
\mathbf{0} & \mathbf{I}
\end{array}\right]\left\{\begin{array}{c}
\mathbf{u}_{\mathbf{Q}} \\
\mathbf{z}
\end{array}\right\}=\lambda_{y}\left[\begin{array}{cc}
\mathbf{A}_{\mathbf{1}} & \mathbf{A}_{\mathbf{2}} \\
\mathbf{I} & \mathbf{0}
\end{array}\right]\left\{\begin{array}{c}
\mathbf{u}_{\mathbf{Q}} \\
\mathbf{z}
\end{array}\right\}
$$

with $\mathbf{I}$ the identity matrix. The propagating wavenumbers are then calculated as :

$$
k_{x}=\frac{\log \left(\lambda_{x}\right)}{-\mathrm{i} d_{x}} \quad \text { and } \quad k_{y}=\frac{\log \left(\lambda_{y}\right)}{-\mathrm{i} d_{y}}
$$

The process of correlating the computed wavenumbers for each frequency and each direction of propagation is exhibited in the next section.

\subsection{Post-processing the results of the solution}

The solutions of the eigenproblem in eq.(10) can be categorized into :

- Propagating and evanescent waves.

- Different wave modes.

In order to distinguish the propagating from evanescent waves an evanescent wave rejection criterion is adopted. Supposing that a calculated wavenumber is in the form $k=\alpha+\beta \mathrm{i}$, we consider that under the condition : $|\alpha| /|\beta|>p$ the wave is propagating, with $p$ an empirical parameter greater that unity. To validate the results, the number of the 
captured propagating waves can be compared to the number of propagating waves when $\mathbf{C}=0$ (thus $\beta=0$ for any propagating wave). A $p=3$ is used throughout this work with no inconsistencies observed.

In order to identify the eigenvalues and eigenvectors corresponding to the same wave mode, each propagating wave mode shape is compared to the whole set of wave mode shapes for each angle and each frequency. The MAC criterion which expresses the correlation of two vectors $\boldsymbol{\Phi}_{\mathbf{i}}$ and $\boldsymbol{\Phi}_{\mathbf{j}}$ was used for this purpose and can be written as :

$$
\operatorname{MAC}=\frac{\left(\boldsymbol{\Phi}_{i}^{T} \overline{\boldsymbol{\Phi}}_{j}\right)\left(\boldsymbol{\Phi}_{j}^{T} \overline{\boldsymbol{\Phi}}_{i}\right)}{\left(\boldsymbol{\Phi}_{i}^{T} \overline{\boldsymbol{\Phi}}_{i}\right)\left(\boldsymbol{\Phi}_{j}^{T} \overline{\boldsymbol{\Phi}}_{j}\right)}
$$

where ${ }^{T}$ stands for the transpose and - for the conjugate of each vector. A high MAC value should firstly be attempted. If no correlated wave modes are found, a lower MAC value is used until all propagating wave modes are correlated to a set of wave types. The wave modes employing out of plane motion (typically the symmetric and the antisymmetric out of plane waves) are then used to conduct the SEA analysis of the structure.

\section{Computation of the energy analysis quantities}

\subsection{Calculation of the modal density}

Using the Courant's formula [29], the modal density of each propagating wave type $w$ can be written for each angle $\phi$ as a function of the propagating wavenumber and its corresponding group velocity $c_{g}$ :

$$
n_{w}(\omega, \phi)=\frac{A k_{w}(\omega, \phi)}{2 \pi^{2}\left|c_{g, w}(\omega, \phi)\right|}
$$

where $A$ is the area of the panel and the group velocity is expressed as :

$$
c_{g}(\omega, \phi)=\frac{\mathrm{d} \omega}{\mathrm{d} k(\omega, \phi)}
$$

The angularly averaged modal density of the structure is eventually given as a function of frequency :

$$
n_{w}(\omega)=\int_{0}^{\pi} n_{w}(\omega, \phi) \mathrm{d} \phi
$$

\subsection{Calculation of the radiation efficiency}

In order to calculate the radiation efficiency $\sigma(k(\omega))$ for each propagating wave type $w$, relations presented in the open bibliography are employed. For continuous structures mode shapes of sinusoidal form can be assumed in order to avoid any FE discretization errors in the solution. The set of asymptotic formulas given in [5] can be used in order to compute $\sigma(k(\omega))$. Within an SEA context, energy equipartition amongst the resonant modes is assumed so that the radiation efficiency is written as :

$$
\sigma_{\text {rad }}(\omega)=\frac{1}{n(\omega)} \int_{0}^{\pi} \sigma(\kappa(\omega, \phi)) n(\omega, \phi) d \phi
$$




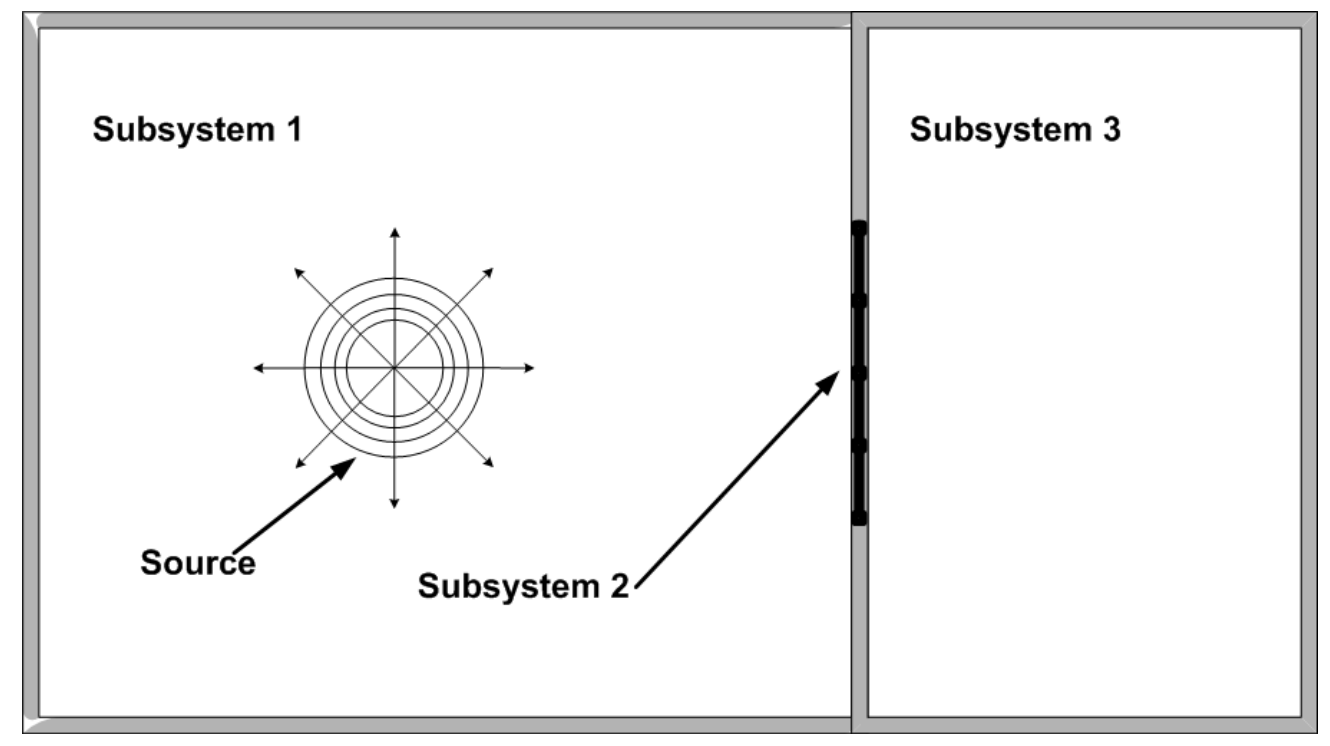

FIGURE 3: The configuration to be modelled by SEA analysis.

For a periodic discontinuous structure the assumption of sinusoidal mode shapes is no longer valid, therefore the radiation efficiency should be calculated directly from the WFEM derived wave mode shapes. The radiation efficiency expression given in [16] can therefore be employed.

\subsection{Calculation of the Sound Transmission Loss (STL) of a panel by an SEA approach}

The STL (or TL) is one of the most important indices of the vibroacoustic performance of a structure. The system to be modelled comprises two reverberant chambers separated by the modelled composite panel attached to a rigid baffle. No flanking transmission is considered in the SEA model. A graphical representation of the modelled subsystems is given in fig.3. The energy balance of the subsystems as it is considered within an SEA approach (see [30]) is illustrated in fig. 4 , in which $E_{1}, E_{3}$ stand for the acoustic energy of the source room and the receiving room respectively and $E_{3}$ for the vibrational energy of the composite panel. Moreover $P_{\text {in }}$ is the injected power in the source room, $P_{1 d}, P_{2 d}$ and $P_{3 d}$ stand for the power dissipated by each subsystem and $P_{13}$ is the non-resonant transmitted power between the rooms. Considering each wave type $w=a, b, c \ldots n$ propagating within the composite panel as a separate SEA subsystem we have :

$$
\begin{aligned}
& P_{12}=\sum_{w=a}^{n} P_{12, w} \\
& P_{23}=\sum_{w=a}^{n} P_{23, w}
\end{aligned}
$$

where $P_{12}$ and $P_{23}$ stand for the power flow between the rooms and the panel.

The STL is defined as : 


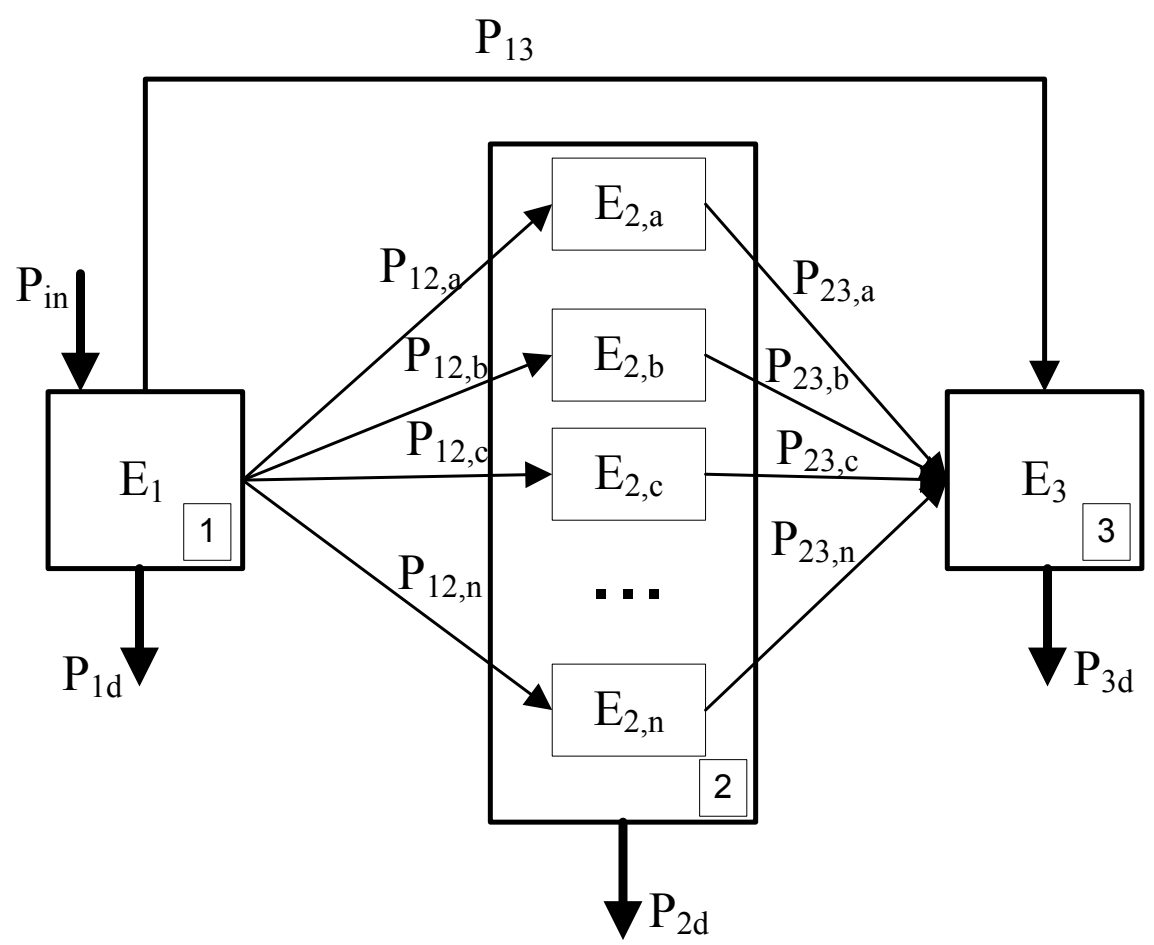

FIGURE 4: A schematic representation of the SEA power exchanges and energies for the modelled system.

$$
\mathrm{STL}=10 \log _{10}\left(\frac{1}{\tau}\right)
$$

where $\tau$ is the transmission coefficient which represents the ratio between the transmitted and the incident sound powers. It can be written as the sum of the resonant and the non-resonant transmission coefficient :

$$
\tau=\frac{P_{23}+P_{13}}{P_{i n c}}=\sum_{w=a}^{n} \frac{P_{23, w}}{P_{i n c}}+\frac{P_{13}}{P_{i n c}}
$$

where $P_{i n c}$ stands for the acoustic power incident on the layered panel, which for a reverberant sound field can be written as :

$$
P_{i n c}=\frac{\left\langle p_{1}^{2}\right\rangle A}{4 \rho c}
$$

where $\left\langle p_{1}^{2}\right\rangle$ the mean-square sound pressure. An attempt to calculate the resonant coefficient for each wave type $w$ is hereby made. Assuming no energy exchanges between different wave types within the structure, the energy balance of a structural wave subsystem can be written as :

$$
P_{12, w}=P_{2 d, w}+P_{23, w}
$$

The power dissipated can be written as : 


$$
P_{2 d, w}=E_{2, w} \omega \eta_{2, w}
$$

with $\eta_{2, w}$ the structural loss factor of the wave type $w$. When the loss factors of each individual layer of the panel are known, the structural loss factor of the entire panel can be computed using the relations provided in [31]. The vibrational energy of the panel due to wave type $w$ can be written as :

$$
E_{2, w}=\rho_{s} A\left\langle v_{w}^{2}\right\rangle
$$

where $\rho_{s}$ is the mass per unit of area, $A$ is the total area of the panel and $\left\langle v_{w}^{2}\right\rangle$ is the mean-square panel vibration velocity due to wave type $w$.

The power flow $P_{12, w}$ can be written using the SEA reciprocity rule, as :

$$
P_{12, w}=\omega \eta_{12, w} n_{1}\left(\frac{E_{1}}{n_{1}}-\frac{E_{2, w}}{n_{2, w}}\right)=\omega \eta_{21, w} n_{2, w}\left(\frac{E_{1}}{n_{1}}-\frac{E_{2, w}}{n_{2, w}}\right)
$$

where $n_{1}, n_{2, w}$ are the modal density of the source room and of the wave type $w$ respectively and $\eta_{21, w}$ the coupling loss factor between the receiving room and the wave type $w$ which can be written as :

$$
\eta_{21, w}=\eta_{23, w}=\frac{\rho c \sigma_{r a d, w}}{\rho_{s} \omega}
$$

Where $\rho$ is the acoustic medium density of the room. The total acoustic energy of the source room can be written as :

$$
E_{1}=\frac{\left\langle p_{1}^{2}\right\rangle V}{\rho c^{2}}
$$

A generally acceptable approximation for the modal density of the source room is made as :

$$
n_{1}=\frac{V_{1} \omega^{2}}{2 \pi^{2} c^{3}}
$$

then the modal energy of the source room can be written as :

$$
\frac{E_{1}}{n_{1}}=\frac{2 \pi^{2} c\left\langle p_{1}^{2}\right\rangle}{\rho \omega^{2}}
$$

Using the SEA reciprocity rule again, the power flow from the composite panel to the receiving room can be written as :

$$
P_{23, w}=\omega \eta_{23, w} n_{2, w}\left(\frac{E_{2, w}}{n_{2, w}}-\frac{E_{3}}{n_{3}}\right)=\omega \eta_{23, w}\left(E_{2, w}-\frac{E_{3} n_{2, w}}{n_{3}}\right)
$$


It is hereby assumed that $n_{3}>>n_{2, w}$ (reasonable for typically sized cavities and especially for medium and high frequencies) and it is also logical that $E_{2, w}>E_{3}$ for an acoustically efficient, out of plane wave. Therefore presuming that $E_{2, w}>>\frac{E_{3} n_{2, w}}{n_{3}}$, eq.(32) can be written as :

$$
P_{23, w}=E_{2, w} \omega \eta_{23, w}
$$

Eventually, after manipulating eq.(23) and eq.(25)-(33) and substituting them into eq.(24) we get :

$$
\frac{\left\langle v_{w}^{2}\right\rangle}{\left\langle p_{1}^{2}\right\rangle}=\frac{2 \pi c^{2} \sigma_{r a d, w} n_{2, w}}{\rho_{s} \omega^{2} A\left(\rho_{s} \omega \eta_{2, w}+2 \rho c \sigma_{r a d, w}\right)}
$$

Using eq.(26),(28),(33),(34),(23) and substituting them into eq.(22) we get the expression for the transmission coefficient of the wave type $w$ :

$$
\tau_{w}=\frac{8 \rho^{2} c^{4} \pi \sigma_{r a d, w}^{2} n_{2, w}}{\rho_{s} \omega^{2} A\left(\rho_{s} \omega \eta_{2, w}+2 \rho c \sigma_{r a d, w}\right)}
$$

The total transmission coefficient of the panel $\tau$ can now be written as :

$$
\tau=\sum_{w=a}^{n} \tau_{w}+\frac{P_{13}}{P_{i n c}}
$$

The non resonant transmission coefficient $\tau_{n r}=P_{13} / P_{\text {inc }}$ for a diffused acoustic field can be written as in [17] :

$$
\frac{P_{13}}{P_{\text {inc }}}=\frac{\int_{0}^{2 \pi} \int_{0}^{\theta_{\max }} \frac{4 Z_{0}^{2} \sigma(\theta, \phi, \omega) \sin \theta \cos ^{2} \theta}{\left|i \omega \rho_{s}+2 Z_{0}\right|^{2}} \mathrm{~d} \theta \mathrm{d} \phi}{\pi\left(1-\cos ^{2} \theta_{\text {max }}\right)}
$$

in which $\theta$ and $\phi$ are the incidence angle and the direction angle of the acoustic wave respectively, and $Z_{0}=$ $\rho c / \cos \theta$ is the acoustic impedance of the medium. The term $\theta_{\max }$ stands for the maximum incidence angle, accounting for the diffuseness of the incident field. It is considered that $\theta_{\max }=p i / 2$ for all the results presented in the current work. The term $\sigma(\theta, \phi, \omega)$ is the corrected radiation efficiency term. It is used in order to account for the finite dimensions of the panel by accounting for the radiation of the mass controlled non-resonant modes, and it is calculated using a spatial windowing correction technique presented in [32]. In eq.(36) the total transmission coefficient of the layered panel is expressed merely as a function of its SEA quantities and independently of the room dimensions and modal energies.

\section{Numerical examples}

In this section numerical applications of the approach described above will be presented. In order to validate the proposed models, four structures were chosen to be computed; the first being a thin stratified orthotropic composite panel. Subsequently, two honeycomb sandwich structures as well as a particularly thick sandwich panel comprising a soft core are modelled. It should be noted that experimental results for the response of layered panels under a 
TABLE 1: Mechanical properties of materials

\begin{tabular}{ccccccc}
\hline \hline Material I & Material II & Material III & Material IV & Material V & Material VI & Material VII \\
\hline$\rho=9740 \mathrm{~kg} / \mathrm{m}^{3}$ & $\rho=1600 \mathrm{~kg} / \mathrm{m}^{3}$ & $\rho=160 \mathrm{~kg} / \mathrm{m}^{3}$ & $\rho=1550 \mathrm{~kg} / \mathrm{m}^{3}$ & $\rho=110.44 \mathrm{~kg} / \mathrm{m}^{3}$ & $\rho=629.9 \mathrm{~kg} / \mathrm{m}^{3}$ & $\rho=16 \mathrm{~kg} / \mathrm{m}^{3}$ \\
$v_{x y}=0.028$ & $v_{x y}=0.15$ & $v_{x y}=0.15$ & $v_{x y}=0.3$ & $v_{x y}=0.45$ & $v_{x y}=0.15$ & $v_{x y}=0.34$ \\
$v_{x z}=0.001$ & $v_{x z}=0.15$ & $v_{x z}=0.15$ & $v_{x z}=0.3$ & $v_{x z}=0.45$ & $v_{x z}=0.15$ & $v_{x z}=0.34$ \\
$v_{y z}=0.434$ & $v_{y z}=0.15$ & $v_{y z}=0.15$ & $v_{y z}=0.3$ & $v_{y z}=0.45$ & $v_{y z}=0.15$ & $v_{y z}=0.34$ \\
$E_{x}=2023.7 \mathrm{GPa}$ & $E_{x}=49 \mathrm{GPa}$ & $E_{x}=0.25 \mathrm{GPa}$ & $E_{x}=48 \mathrm{GPa}$ & $E_{x}=0.1448 \mathrm{GPa}$ & $E_{x}=8.3 \mathrm{GPa}$ & $E_{x}=0.0083 \mathrm{GPa}$ \\
$E_{y}=31375 \mathrm{GPa}$ & $E_{y}=49 \mathrm{GPa}$ & $E_{y}=0.25 \mathrm{GPa}$ & $E_{y}=48 \mathrm{GPa}$ & $E_{y}=0.1448 \mathrm{GPa}$ & $E_{y}=8.3 \mathrm{GPa}$ & $E_{y}=0.0083 \mathrm{GPa}$ \\
$E_{z}=38000 \mathrm{GPa}$ & $E_{z}=49 \mathrm{GPa}$ & $E_{z}=0.25 \mathrm{GPa}$ & $E_{z}=48 \mathrm{GPa}$ & $E_{z}=0.1488 \mathrm{GPa}$ & $E_{z}=8.3 \mathrm{GPa}$ & $E_{z}=0.0083 \mathrm{GPa}$ \\
$G_{x y}=888.79 \mathrm{GPa}$ & $G_{x y}=21.3 \mathrm{GPa}$ & $G_{x y}=0.11 \mathrm{GPa}$ & $G_{x y}=18.1 \mathrm{GPa}$ & $G_{x y}=0.05 \mathrm{GPa}$ & $G_{x y}=3.6 \mathrm{GPa}$ & $G_{x y}=0.0031 \mathrm{GPa}$ \\
$G_{y z}=888.79 \mathrm{GPa}$ & $G_{y z}=21.3 \mathrm{GPa}$ & $G_{y z}=0.09 \mathrm{GPa}$ & $G_{y z}=2.76 \mathrm{GPa}$ & $G_{y z}=0.05 \mathrm{GPa}$ & $G_{y z}=3.6 \mathrm{GPa}$ & $G_{y z}=0.0031 \mathrm{GPa}$ \\
$G_{x z}=888.79 \mathrm{GPa}$ & $G_{x z}=21.3 \mathrm{GPa}$ & $G_{x z}=0.14 \mathrm{GPa}$ & $G_{x z}=2.76 \mathrm{GPa}$ & $G_{x z}=0.05 \mathrm{GPa}$ & $G_{x z}=3.6 \mathrm{GPa}$ & $G_{x z}=0.0031 \mathrm{GPa}$ \\
\hline
\end{tabular}

reverberant acoustic field are rare to find in the open bibliography. Consequently, the available options of composite structures to be modelled while concurrently having test data to compare with were not a lot. The mechanical static characteristics of each material used for the validation process are mentioned in Table 1, in which $\rho$ is the density of the material, $E_{i}$ the Young modulus in direction $i, G_{i j}$ stands for the shear modulus in direction $j$ on the plane whose normal is in direction $i$ and $v_{i j}$ for the Poisson's ratio that corresponds to a contraction in direction $j$ when an extension is applied in direction $i$. The FE modelling was conducted within ANSYS 12.1 software. The segments were modelled using the linear eight-node ANSYS SOLID45 element which comprises a 3D displacement field and three DoF per node (translations in the $x, y$, and $z$ directions). A minimum number of one elements per layer was used in order to minimize the numerical errors produced by the $\mathbf{D}$ matrix computation (see [33]). In order to avoid any interpolation errors, it was also ensured that the elements maximum dimensions were inferior to one tenth of the minimum structural wavelength propagating within the frequency range of the analysis.

\subsection{Validation on an orthotropic thin monolithic structure}

The wavenumbers for the bending, shear and membrane wave types were identified for a thin, stratified type structure for propagation towards $x$ direction. The panel is made of material I. Its thickness is equal to $h=0.5 \mathrm{~mm}$ and its dimensions are $1.4 \mathrm{~m} \times 0.9 \mathrm{~m}$. The resulting dispersion curves are presented in fig.5, and the results are compared with the CPT analytic solutions (see [1]). Excellent accordance is observed for the shear and the membrane wave types. Concerning the flexural wave type, the WFE predictions are very much in agreement with the analytical formula even for the high frequency range. To give an idea for the discrepancy between the two models, a difference of $0.09 \%$ is observed at $10 \mathrm{kHz}$. Under an acoustic excitation, antisymmetric wave modes are responsible for the transmission of the vast majority of energy through the structure, therefore they will be the main wave type to be considered in the SEA analysis.

The flexural wavenumbers are presented as a function of direction and frequency in fig.6. The directional dependence of the wavenumber within a highly orthotropic panel is observed. It is known that each mode of the panel corresponds to an exact set of wavenumbers depending on the panels boundary conditions. For a simply supported 


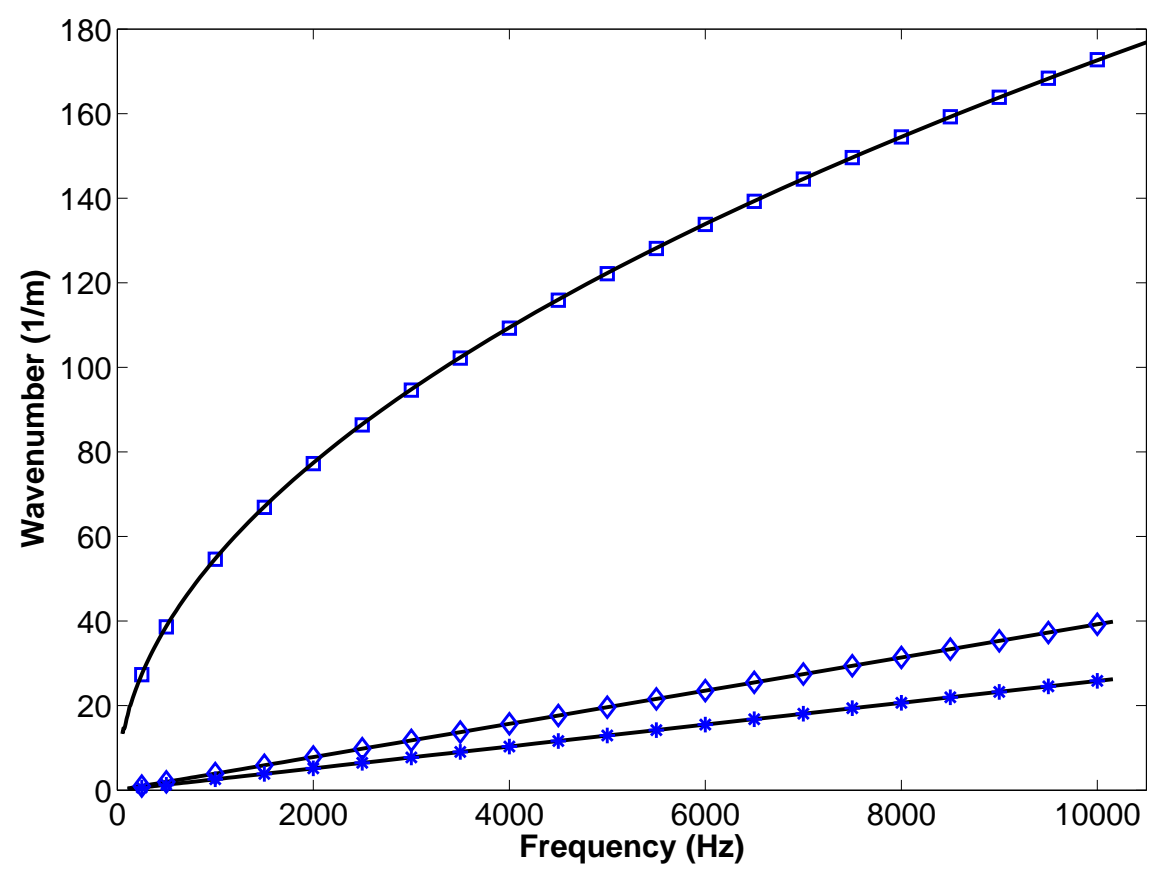

FIGURE 5: Comparison of the WFEM calculated dispersion curves towards $x$ direction (-) with CPT analytical formulas for : flexural ( $\square$ ), shear ( $\diamond)$ and membrane $(*)$ propagating wavenumbers for a thin orthotropic panel

panel this set is : $k_{x}=m \pi / L_{x}$ and $k_{y}=n \pi / L_{y}$ where $m, n$ are integers and $L_{i}$ the length and width of the panel. The resonance frequency for the $m, n$ flexural mode of the panel can be sought by conducting a 2D linear interpolation of this $k_{x}, k_{y}$ set to the $\omega, k_{x}, k_{y}$ relationship as depicted in fig.6.

The modal density of the monolithic orthotropic panel is subsequently calculated using the antisymmetric wave propagation characteristics presented in fig.6 and eq.(18). Small discrepancies are observed between the values calculated using the WFEM and the CPT because of the fact that the latter approach does not account for the shear effects within the laminate. As expected, the two predictions are very similar for the low frequency range. The largest divergence for the two predictions is $4.1 \%$ and is observed at the highest frequency range of the analysis. It is seen that taking into account for the shear effects within the laminate becomes important as frequency increases.

The radiation efficiency of the monolithic orthotropic panel is calculated using the Leppington's asymptotic and transition formulas. The results are shown in fig. 8 . The beginning of the coincidence range (approximately $2 \mathrm{kHz}$ ) is marked by an intensive increase of the radiation efficiency, which continues rising steadily throughout the coincidence region. The end of the coincidence range (approximately $9.5 \mathrm{kHz}$ ) is marked with a peak of the radiation efficiency curve. Fluctuations of the curve throughout the coincidence range were expected and are discussed in [5]. The individual modal radiation efficiencies are also calculated using the formulas given in [34] and presented in the same figure. Each mode is separately attributed to a different category depending on its individual characteristics namely the directional wavenumbers $k_{x}$ and $k_{y}$ and its resonance frequency. It can be clearly observed that the corner modes are the less radiating ones. On the other hand, the surface modes are particularly efficient due to the fact that the entirety 


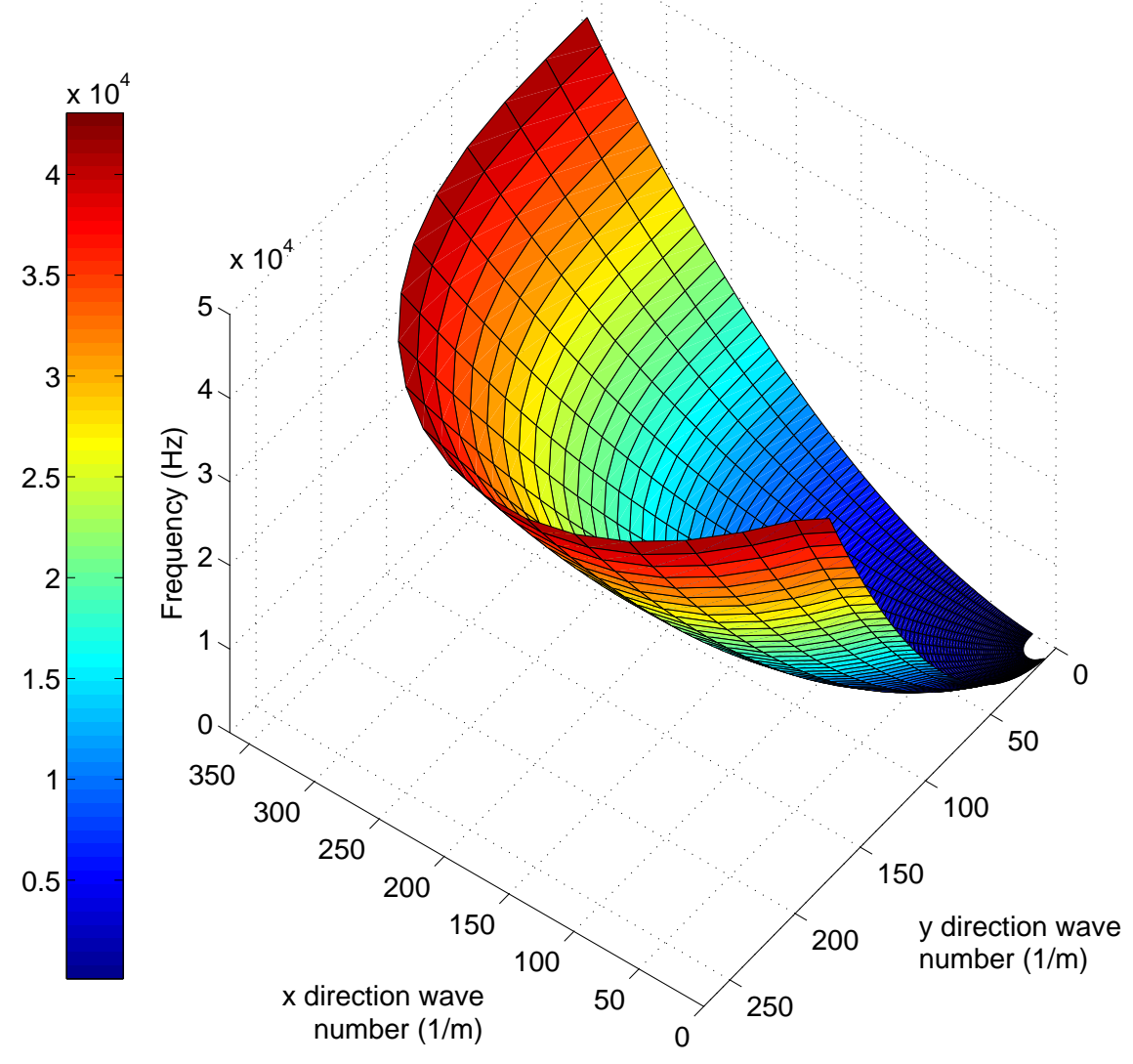

FIGURE 6: 3-dimensional view of the bending propagating wavenumbers within the stratified structure as a function to direction of propagation and frequency

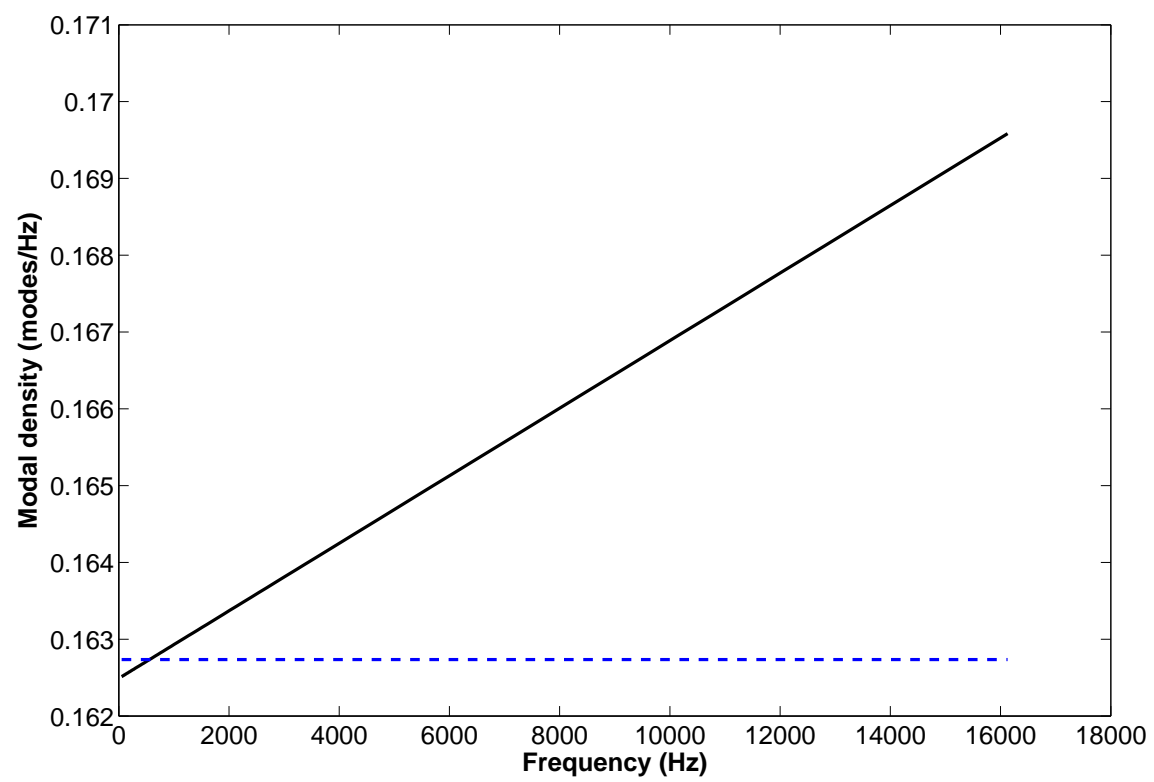

FIGURE 7: Comparison of the predicted modal density for a monolithic panel : present approach (-), CPT (- -) 


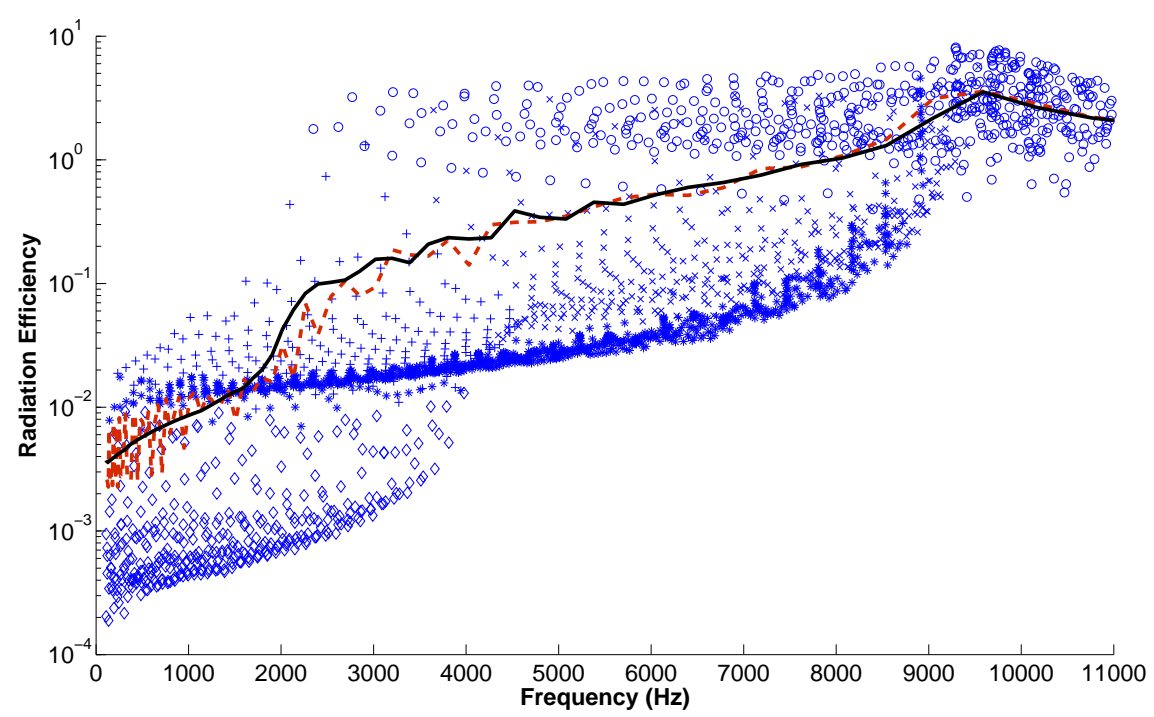

FIGURE 8: Comparison of the radiation efficiency of a monolithic orthotropic panel using : the present methodology (-), modal radiation formulas in [34] (surface modes 'o', x-edge modes '+', y-edge modes '*', x-y edge modes 'x', corner modes ' $\diamond$ ), frequency averaged radiation efficiency according to [35] (- -)

of the surface of the panel radiates in the acoustic medium. The modal radiation values are frequency averaged as suggested in [35]. The result is presented in the same figure. In the low frequency range the averaged result presents an intense fluctuation due to the small number of modes taken into account within each frequency band (1/12 octave frequency bands). Above $2.5 \mathrm{KHz}$, the surface modes hit in, drastically increasing the radiation efficiency of the panel. Very good agreement is observed comparing with the Leppington's formulas predictions.

The STL of the orthotropic thin panel under a reverberant acoustic field is presented in fig.9. On the same figure experimental data for the same quantity, published in [6] are shown. The results of an asymptotic TL calculation model presented in [6] are also compared in the same figure. Excellent agreement between the experimental and predicted results is observed for the sub-coincident frequency range as well as for the whole coincidence range. The radiation efficiency is not overestimated -in contrast to the asymptotic model- at the beginning of the coincidence area. The fluctuations of the TL curve throughout the coincidence range are due to the radiation efficiency formulas used and are discussed in [5]. A structural loss factor equal to 0.01 is used. The discrepancy between the numerical prediction and the experimental results at the end of the coincidence range can either be attributed to an overestimation of the radiation efficiency or to a higher structural damping coefficient (probably due to large deflections) for the panel in this frequency range. Fast convergence of the predicted values towards the experimental data is observed in the post-coincident frequency range.

\subsection{Validation on honeycomb sandwich panels}

A sandwich panel is subsequently considered. It comprises a core made of material $\mathrm{V}$ and facesheets made of material IV. The thickness of the core is equal to $12.7 \mathrm{~mm}$ while the thickness of the facesheets is equal to $1.2 \mathrm{~mm}$. The 


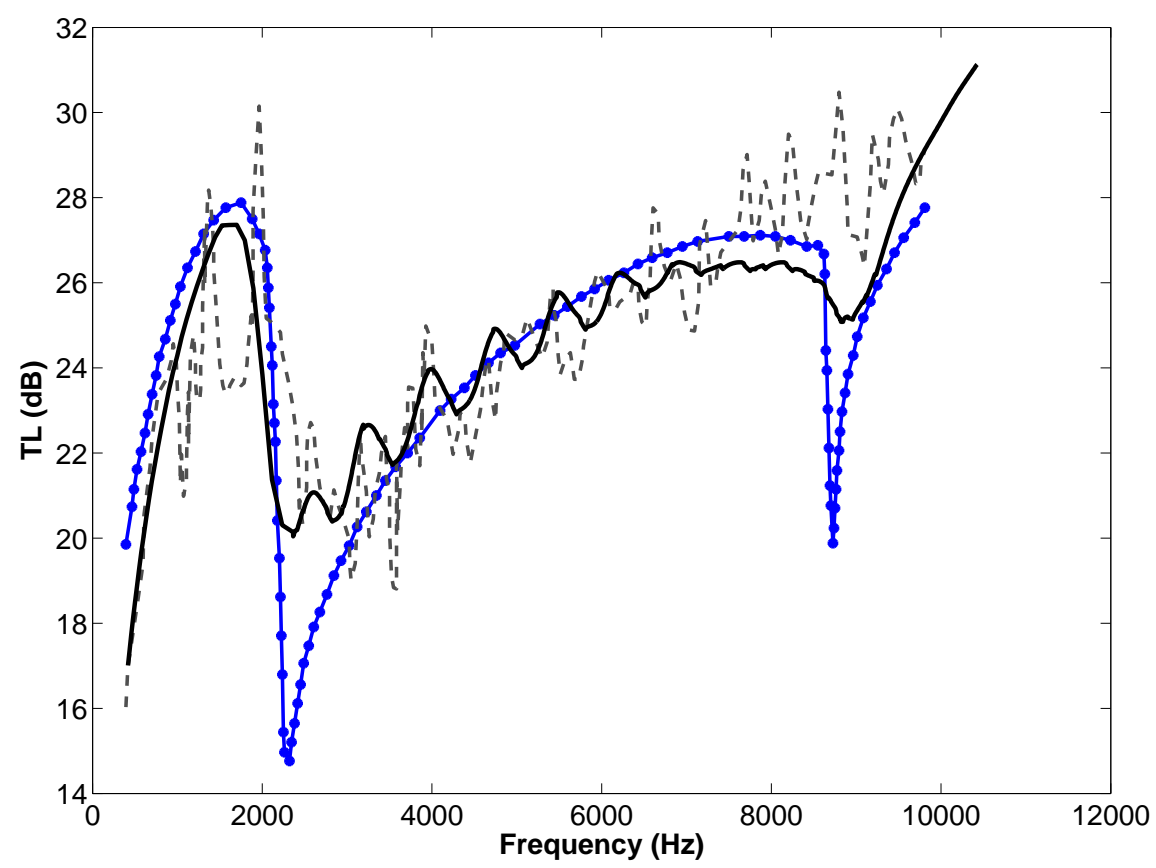

FIGURE 9: Comparison of the diffused field TL of an orthotropic thin panel : present methodology (-), model in [6] (*), experimental results in [6] $(--)$

dimensions of the panel are equal to $1.37 \mathrm{~m} \times 1.65 \mathrm{~m}$. The calculated antisymmetric wavenumbers of the panel in $x$ direction are presented in fig.10. The results in [17] for the same panel using a LW approach are exhibited in the same figure and are in excellent agreement with the presented approach. The frequency ranges of the dynamic behaviour of the sandwich panel are clearly distinguished (see also [12]). A low-frequency region where the panel vibrates as a whole, an intermediate region where the shear stiffness of the panel dominates its vibrational behaviour and a highfrequency region where the flexural wavenumber for the panel is converging to the wavenumber of each facesheet vibrating separately, loaded with half of the core mass. The dispersion relation predicted by a Mindlin type model proposed for thick sandwich panels in [36] which takes into account the shear deformation of the panel is depicted in the same figure. The Mindlin theory diverges quickly when the sandwich panel enters the shear deformation dominated region. As a result a misguiding coincidence frequency for the antisymmetric wave $\left(f_{c 1}=560 \mathrm{~Hz}\right)$ is predicted, which is far from the one predicted by the WFEM $\left(f_{c 1}=1190 \mathrm{~Hz}\right)$. At higher frequencies the Mindlin model further diverges, having a difference of $250 \%$ at $40 \mathrm{kHz}$. The asymptotic Kurtze and Watters (KW) model implemented as presented in [37] is also shown in the same figure. It is seen that the KW prediction agrees asymptotically with the WFEM model, with the three regions of the panel clearly distinguished. However discrepancies between the two models occur at the transition regions of the $\mathrm{KW}$ model.

In order to validate the WFEM predicted values for the modal density of a sandwich panel, a structure presented in [38] was modelled. It comprises a honeycomb foam core made of material III and facesheets made of material II. The thickness of the core is equal to $6.35 \mathrm{~mm}$ while the thickness of the facesheets is equal to $0.5 \mathrm{~mm}$. The dimensions of 


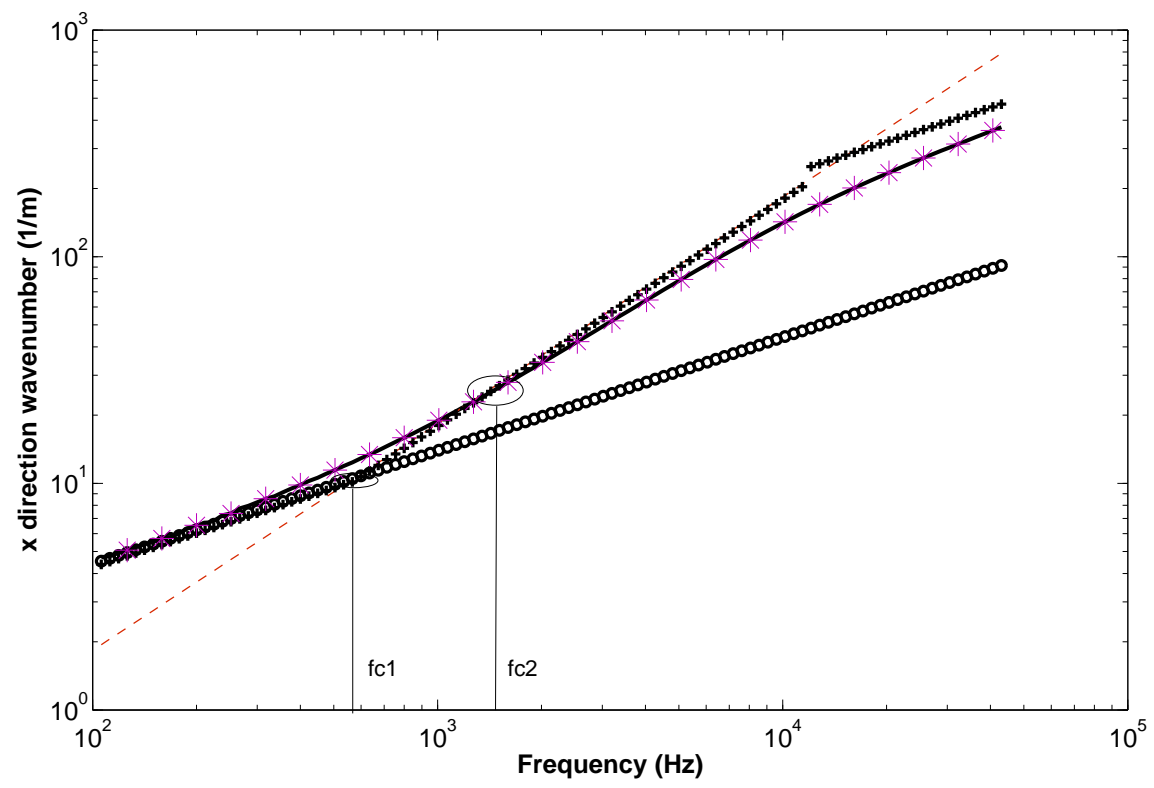

FIGURE 10: Comparison of the wavenumber in $\mathrm{x}$ direction for a sandwich panel : present methodology (-), results in [17] (*), a Mindlin type model (o), Kurtze-Watters model (+), acoustic wavenumber (--)

the panel are equal to $1.12 \mathrm{~m} \times 0.62 \mathrm{~m}$, but were reduced to $0.84 \mathrm{~m} \times 0.42 \mathrm{~m}$ when the panel was fixed to the baffle. The results of the modal density are shown in fig.11. An excellent agreement between the calculations and the experimental measurements is observed. Within the lower frequency range the present approach seems to correctly predict the mean value around which the measured modal density is dispersed.

The radiation efficiency of the honeycomb foam core sandwich is calculated using the same set of asymptotic formulas as before and is compared to the predictions of the Crocker's model and the experimental data presented in [38]. It is observed that the Leppington's formula combined with the WFEM predictions leads to more accurate results in a broadband frequency range. Furthermore, the used set of formulas does not overestimate the radiation efficiency of the panel at the coincidence range.

Furthermore, the diffused field STL of the foam filled honeycomb sandwich structure is calculated. The result is depicted in fig. 13 along with experimental data presented in [38]. The results demonstrate a very good correlation. The low-frequency response seems to be very well simulated. The coincidence frequency band is very well predicted and above coincidence discrepancies of less than $2 \mathrm{~dB}$ are observed between the experimental results and the SEA models. Higher experimental STL values around the coincidence frequency are probably due to an increase of damping for the panel. In the same figure the results of the SEA model presented in [38] are also shown. The discrepancies between the two SEA models are due to the better prediction of the radiation efficiency by the current approach and the spatial windowing correction hereby used. Above coincidence, the results of the two SEA models are very well correlated. 


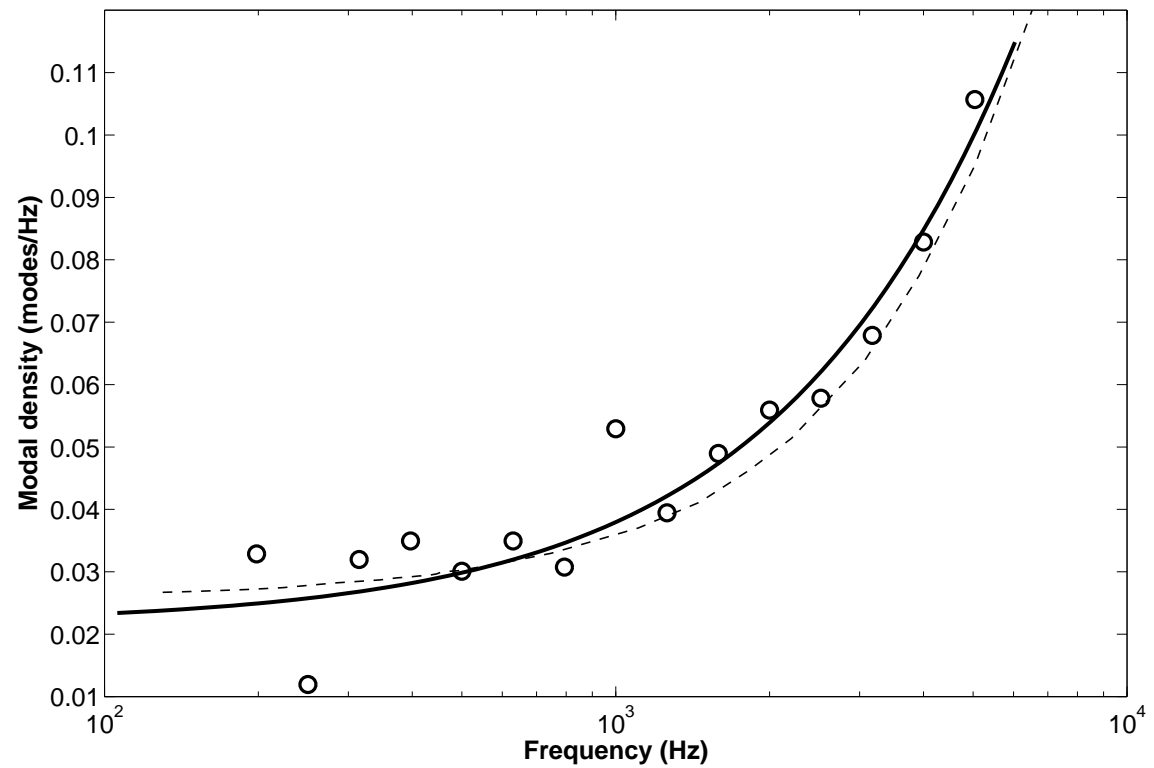

FIGURE 11: Comparison of the predicted modal density for a sandwich panel : present approach (-), model in [39] (--), experimental results in [38] (o)

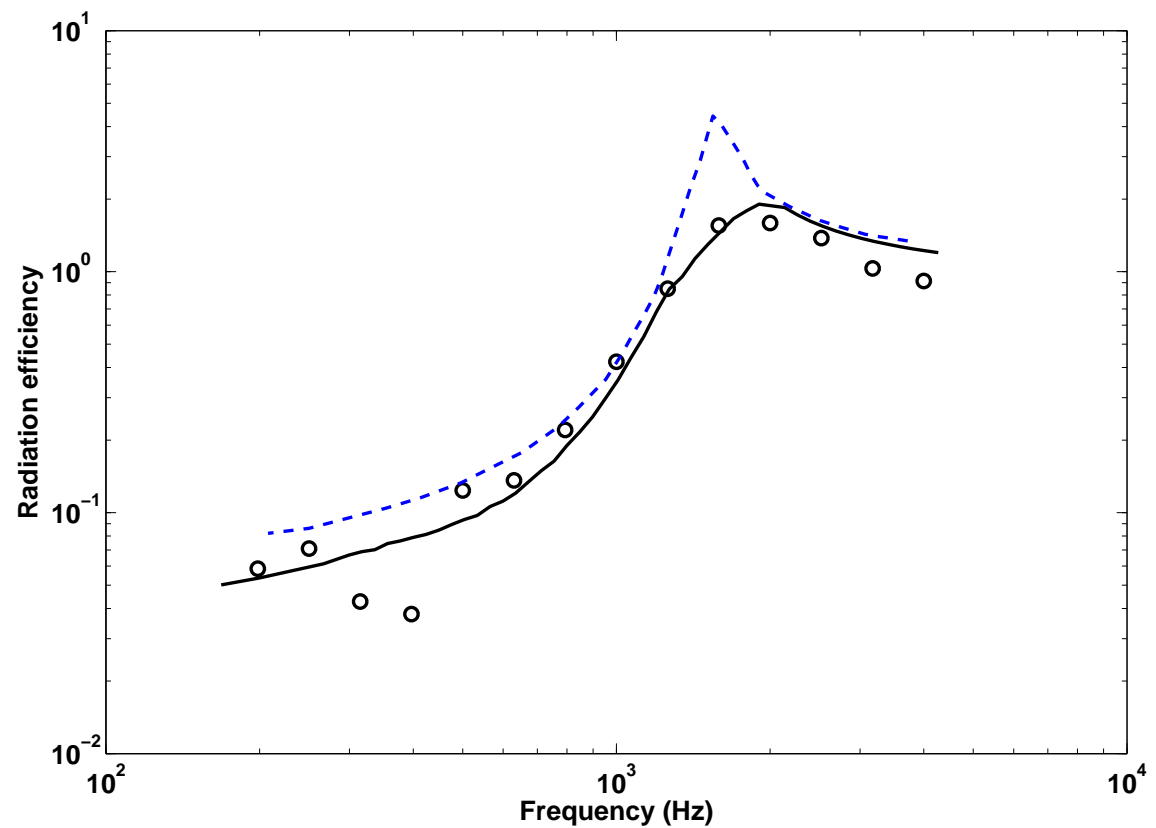

FIGURE 12: Comparison of the predicted radiation efficiency for a sandwich panel : Leppington's set of formulas (-), model in [38] (- -), experimental results in [38] (o) 


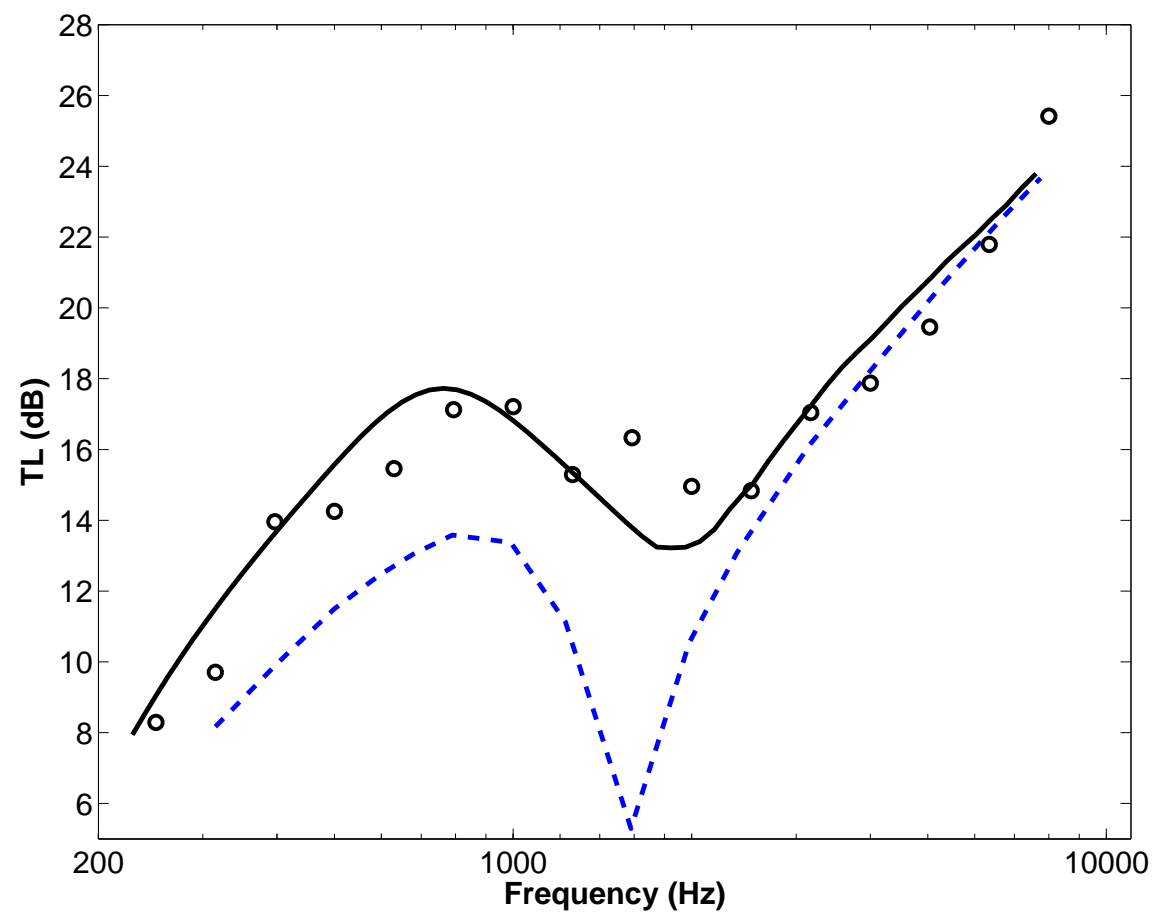

FIGURE 13: Comparison of the diffused field TL of a sandwich panel : present methodology (-), model in [38] (--), experimental results in [38] (o)

\subsection{Validation on a thick layered panel}

For layered panels comprising a thick and soft core, the dilatational motion of the soft layer (also referred to as symmetric motion) can contribute significantly to the resonant acoustic transmission of the panel (see [8]). In order to exhibit the robustness of the presented approach considering the modelling of arbitrarily thick panels a sandwich structure comprising a $38.1 \mathrm{~mm}$ core made of material VII, coupled to $6.35 \mathrm{~mm}$ thick facesheets made of material VI is hereby modelled. The WFEM computed phase velocities for the propagating, out of plane wave motions are shown in fig.14 and are compared to predictions of an analytic HSDT derived model, presented in [12]. Excellent correlation is observed between the two predictions.

A cut on frequency is observed for the symmetric motion above which the wave starts propagating within the panel. Below this cut on frequency the dilatational motion is not expected to influence the transmission coefficient of the panel due to the particularly low modal density of the wave mode. A lock-up of the symmetric and the antisymmetric wave motion is observed for higher frequencies. The total transmission coefficient of the panel is calculated using eq.(36) and the STL of the structure is exhibited in fig.15 along with experimental results provided in [12].

Very good correlation between the computed values and the measurements is observed below the coincidence range. It is noted that the acoustic coincidence frequency for the antisymmetric motion of the panel occurs at $2900 \mathrm{~Hz}$. At $2000 \mathrm{~Hz}$ the impact of the dilatational motion of the panel on its TL becomes evident with the values presenting a sudden decrease of $14 \mathrm{~dB}$ at the cut on frequency of the symmetric wave. The coincidence range is extended up 


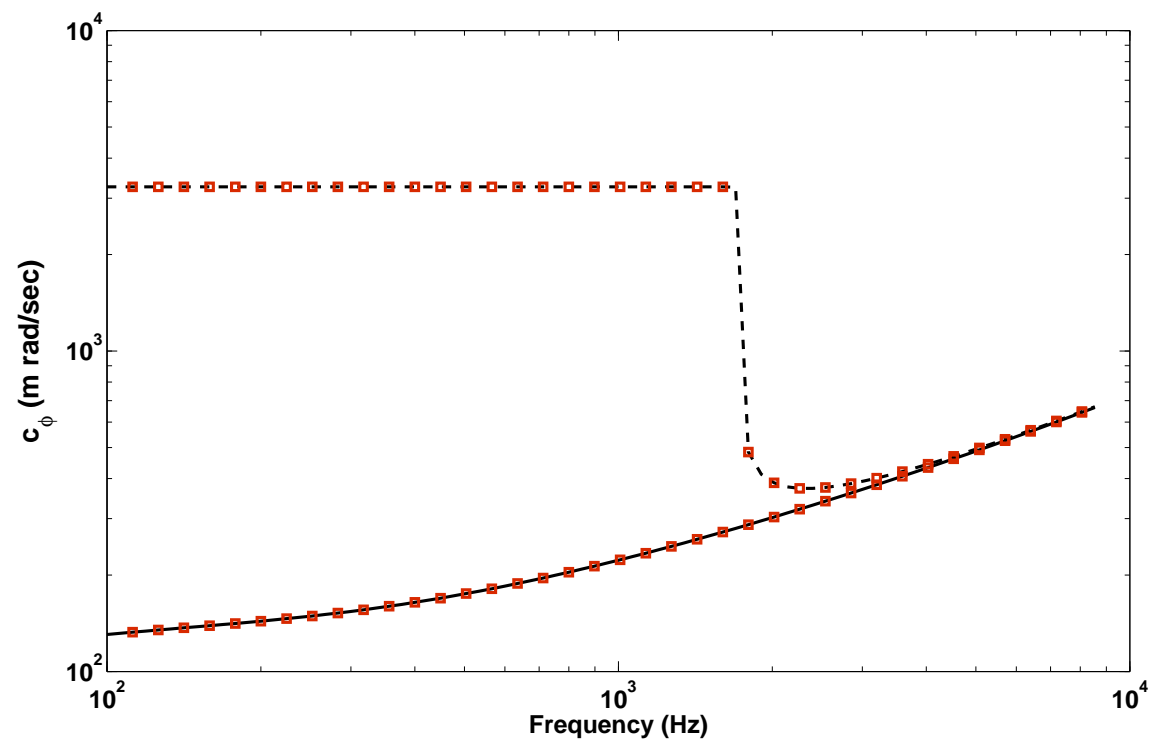

FIGURE 14: Predicted wavenumbers for the out of plane motion of the thick panel : WFEM antisymmetric (-), WFEM symmetric (--), results in [12] ( $\square)$

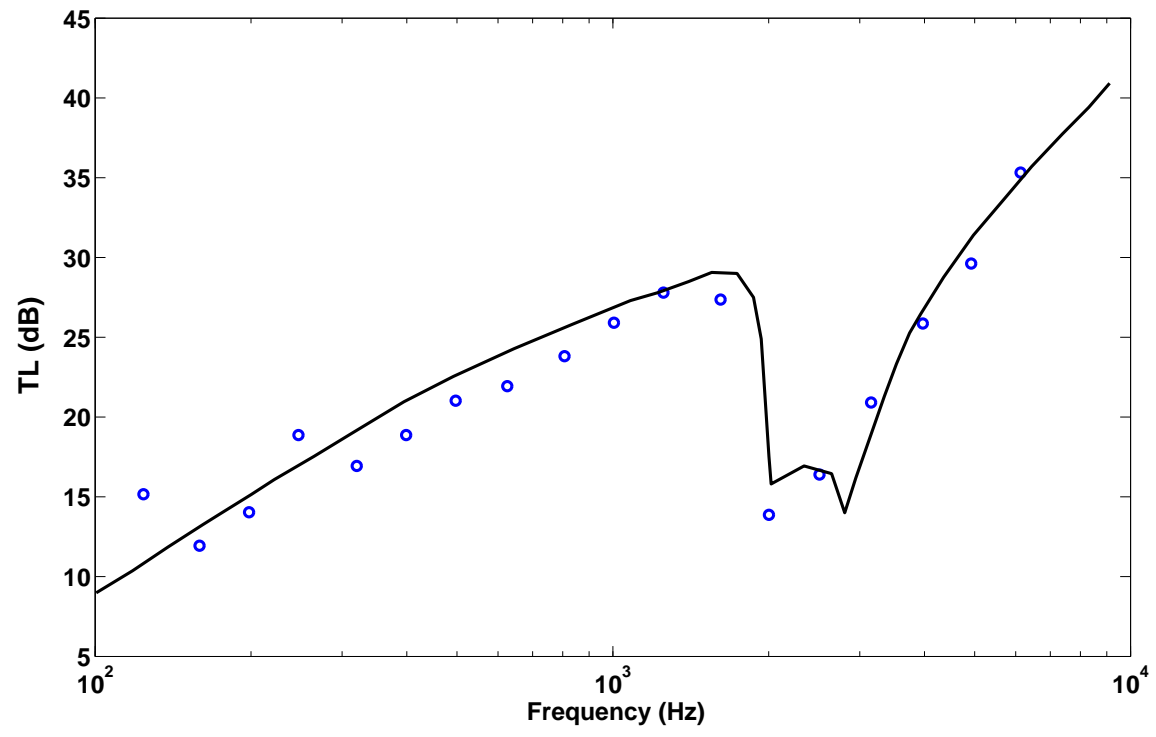

FIGURE 15: Comparison of the diffused field TL of the thick sandwich panel : present approach (-), experimental results in [12, 40] (o) 


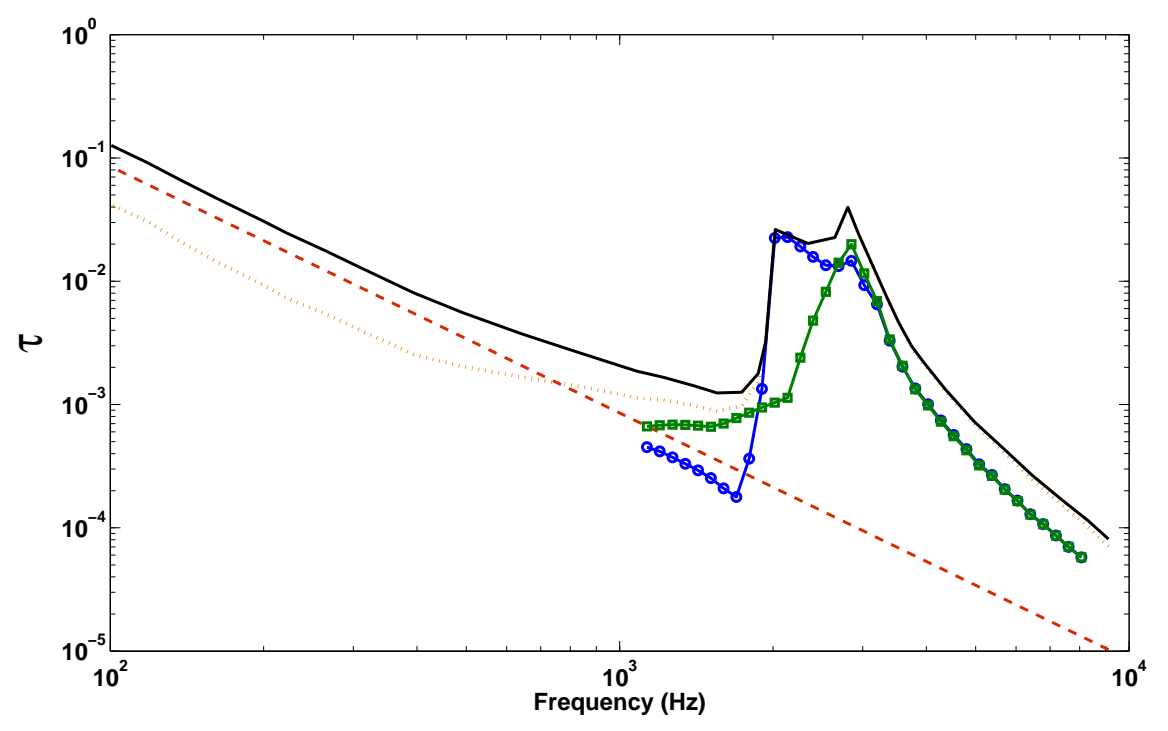

FIGURE 16: Contribution of each transmission path to the transmission coefficient of the panel : total transmission coefficient (-), non-resonant transmission coefficient (- ), resonant transmission coefficient $(\cdots)$, symmetric motion transmission coefficient (o), antisymmetric motion transmission coefficient $(\square)$

to $3000 \mathrm{~Hz}$ because of the antisymmetric coincidence phenomenon and because of the fact that the symmetric phase velocity remains very close to the one of the acoustic medium. A closer look to the contribution of each acoustic transmission path to the total transmission coefficient is shown in fig.16.

The mass controlled phenomena dominate the low frequency range of the total transmission coefficient. Approaching the coincidence range, the resonant contribution becomes the most important one. The symmetric motion of the panel dominates its acoustic transmission at the dilatational motion cut on, while close to the antisymmetric coincidence frequency both wave motions contribute to the transmission. In the post coincidence frequency range the contribution of the two wave motions is almost equal due to fact that the corresponding phase velocities are very close. It is therefore shown that thick and heavy layers do not always reduce the acoustic transmission of a panel because of the fact that they also reduce its dilatational cut on frequency. It is observed that the effect of the symmetric motion on the acoustic transparency of a panel may not be neglected. Care has to be taken therefore when designing an insulating structure for the existence of more than one out of plane propagating wave modes.

\section{Conclusions}

The modelling of the vibroacoustic behaviour of composite layered structures of arbitrary layering and orthotropy was conducted through a wave-context SEA approach. Summarizing the most important points of the presented work : 1) A description of the formulation of the WFE approach was given. The process followed for post-processing the results of the resulting eigenproblem was described. 2) The WFEM was applied to composite, arbitrarily layered panels in order to predict their dispersion characteristics. The predictions were successfully compared to bibliographic 
results. The accuracy of the WFEM compared to analytical predictions, especially with regard to predicting the correct coincidence frequency of the structures is of great importance for an accurate vibroacoustic analysis. 3) The main SEA quantities, namely the modal density and the radiation efficiency of the panels were computed following the calculation of their dispersion characteristics. The calculation was done using analytic formulas in a wave context. The results showed an excellent correlation between experimental data and the current approach. 4) For the calculation of the STL of the panels a SEA approach was adopted. A formula for computing the STL derived directly by the characteristics of the structures, with no dependence on the room properties was given. A generally very good agreement between experimental measurements and the predictions of the presented method was observed throughout the frequency band, validating the effectiveness and the robustness of the later. 5) The symmetric and the antisymmetric wave motion within a thick layered panel was well predicted. It was shown that adding mass to a panel does not always reduce its acoustic transmission and that the effect of the symmetric motion on the STL especially during its cut-on frequency range should not be neglected.

\section{Acknowledgements}

The authors would like to gratefully acknowledge the ITN Marie Curie project GA-214909 'MID-FREQUENCY :

CAE Methodologies for Mid-Frequency Analysis in Vibration and Acoustics' for the financial support.

\section{Références}

[1] K. F. Graff, Wave motion in elastic solids, Dover Publications, Inc (1991).

[2] J. N. Reddy, Mechanics of laminated composite plates : theory and analysis, volume 1, CRC press Boca Raton, 1997.

[3] Y. Stavsky, Bending and stretching of laminated aelotropic plates, Proc.Am.Soc.Civil Engrs. 87 (1961) 31-56.

[4] J. Whitney, N. Pagano, Shear deformation in heterogeneous anisotropic plates, A Appl Mech Trans ASME 37 Ser E (1970) 1031-6.

[5] F. G. Leppington, E. G. Broadbent, K. H. Heron, Acoustic radiation efficiency of rectangular panels, in : Proceedings of The Royal Society of London, Series A : Mathematical and Physical Sciences, volume 382, pp. 245-71.

[6] F. G. Leppington, K. H. Heron, E. G. Broadbent, Resonant and non-resonant transmission of random noise through complex plates, Proceedings of the Royal Society A : Mathematical, Physical and Engineering Sciences 458 (2002) 683-704.

[7] G. Kurtze, B. G. Watters, New wall design for high transmission loss or high damping, J.Acoust.Soc.Am. 31 (1959) 739-48.

[8] C. L. Dym, M. A. Lang, Transmission of sound through sandwich panels, Journal of the Acoustical Society of America 56 (1974) $1523-32$.

[9] R. D. Ford, P. Lord, A. W. Walker, Sound transmission through sandwich constructions, Journal of Sound and Vibration 5 (1967) 9-21.

[10] J. A. Moore, R. H. Lyon, Sound transmission loss characteristics of sandwich panel constructions, Journal of the Acoustical Society of America 89 (1991) 777-91.

[11] V. Sokolinsky, S. Nutt, Consistent higher-order dynamic equations for soft-core sandwich beams, AIAA J 42 (2004) 374-82.

[12] T. Wang, S. Li, S. Rajaram, S. R. Nutt, Predicting the sound transmission loss of sandwich panels by statistical energy analysis approach, Journal of Vibration and Acoustics, Transactions of the ASME 132 (2010) 0110041-.

[13] T. Wang, V. S. Sokolinsky, S. Rajaram, S. R. Nutt, Assessment of sandwich models for the prediction of sound transmission loss in unidirectional sandwich panels, Applied Acoustics 66 (2005) 245-62.

[14] S. Finnveden, Evaluation of modal density and group velocity by a finite element method, Journal of Sound and Vibration 273 (2004) 51-75. 
[15] E. Barbieri, A. Cammarano, S. De Rosa, F. Franco, Waveguides of a composite plate by using the spectral finite element approach, JVC/Journal of Vibration and Control 15 (2009) 347-67.

[16] V. Cotoni, R. S. Langley, P. J. Shorter, A statistical energy analysis subsystem formulation using finite element and periodic structure theory, Journal of Sound and Vibration 318 (2008) 1077-108.

[17] S. Ghinet, N. Atalla, Vibro-acoustic behaviors of flat sandwich composite panels, Transactions of the Canadian Society for Mechanical Engineering 30 (2006) 473-93.

[18] S. Ghinet, N. Atalla, H. Osman, The transmission loss of curved laminates and sandwich composite panels, Journal of the Acoustical Society of America 118 (2005) 774-90.

[19] S. Ghinet, N. Atalla, Modeling thick composite laminate and sandwich structures with linear viscoelastic damping, Computers and Structures 89 (2011) 1547-61.

[20] D. J. Mead, A general theory of harmonic wave propagation in linear periodic systems with multiple coupling, Journal of Sound and Vibration 27 (1973) 235-60.

[21] B. Mace, D. Duhamel, M. Brennan, L. Hinke, Finite element prediction of wave motion in structural waveguides, Journal of the Acoustical Society of America 117 (2005) 2835-43.

[22] J. . Mencik, M. N. Ichchou, Multi-mode propagation and diffusion in structures through finite elements, European Journal of Mechanics, A/Solids 24 (2005) 877-98.

[23] B. Mace, E. Manconi, Modelling wave propagation in two-dimensional structures using finite element analysis, Journal of Sound and Vibration 318 (2008) 884-902.

[24] L. Houillon, Modlisation vibratoire des carrosseries automobiles en moyennes et hautes frquences, (Vibrational modelling of automotive chassis, for medium and high frequenceis), PhD Thesis, Ecole Centrale de Lyon (1999).

[25] M. N. Ichchou, J. Berthaut, M. Collet, Multi-mode wave propagation in ribbed plates : Part I, wavenumber-space characteristics, International Journal of Solids and Structures 45 (2008) 1179-95.

[26] M. N. Ichchou, J. Berthaut, M. Collet, Multi-mode wave propagation in ribbed plates : Part II : Predictions and comparisons, International Journal of Solids and Structures 45 (2008) 1196-216.

[27] D. Chronopoulos, B. Troclet, M. Ichchou, J. Lain, A unified approach for the broadband vibroacoustic response of composite shells, Composites Part B : Engineering 43 (2012) 1837-46.

[28] F. Tisseur, K. Meerbergen, The quadratic eigenvalue problem, SIAM Review 43 (2001) 235-86.

[29] R. Courant, D. Hilbert, Methods of mathematical physics, vol. 1, New York : John Wiley (1989).

[30] R. Lyon, R. DeJong, Theory and application of Statistical Energy Analysis, Butterworth-Heinemann, 1995.

[31] P. Shorter, Wave propagation and damping in linear viscoelastic laminates, Journal of the Acoustical Society of America 115 (2004) $1917-25$.

[32] J. Allard, N. Atalla, Propagation of Sound in Porous Media : Modelling Sound Absorbing Materials 2e, Wiley, 2009.

[33] Y. Waki, B. Mace, M. Brennan, Numerical issues concerning the wave and finite element method for free and forced vibrations of waveguides, Journal of Sound and Vibration 327 (2009) 92-108.

[34] C. E. Wallace, Radiation resistance of a rectangular panel, Journal of the Acoustical Society of America 51 (1972) $946-52$.

[35] J. S. Anderson, M. Bratos-Anderson, Radiation efficiency of rectangular orthotropic plates, Acta Acustica united with Acustica 91 (2005) 61-76.

[36] K. Renji, P. S. Nair, S. Narayanan, Critical and coincidence frequencies of flat panels, Journal of Sound and Vibration 205 (1997) 19-32.

[37] E. B. Davis, Designing honeycomb panels for noise control, AIAA-99-1917 (1999).

[38] R. Zhou, M. J. Crocker, Sound transmission loss of foam-filled honeycomb sandwich panels using statistical energy analysis and theoretical and measured dynamic properties, Journal of Sound and Vibration 329 (2010) 673-86.

[39] B. L. Clarkson, M. F. Ranky, Modal density of honeycomb plates, Journal of Sound and Vibration 91 (1983) 103-18.

[40] S. Narayanan, R. L. Shanbhag, Sound transmission through a damped sandwich panel, Journal of Sound and Vibration 80 (1982) $315-27$. 